\title{
Role of microRNA-218-5p in sevoflurane-induced protective effects in hepatic ischemia/reperfusion injury mice by regulating GAB2/PI3K/AKT pathway
}

\author{
HUI JI, HUI LI, HAIXIA ZHANG and ZHIJUN CHENG \\ Department of Anesthesiology, Xinhua Hospital, \\ Chongming Branch, Shanghai 202150, P.R. China
}

Received November 9, 2020; Accepted May 20, 2021

DOI: $10.3892 / \mathrm{mmr} .2021 .12517$

\begin{abstract}
Hepatic ischemia/reperfusion (I/R) injury (HIRI) often occurs following tissue resection, hemorrhagic shock or transplantation surgery. Previous investigations showed that sevoflurane (Sevo), an inhalation anesthetic, had protective properties against different organ damage in animal models including HIRI. This study is aimed to investigate the underlying mechanisms involved in the protective effects of Sevo on HIRI. The present study results showed that treatment with Sevo improved histologic damage, inflammatory response, oxidative stress and apoptosis after hepatic I/R, indicating the protective role of Sevo against liver I/R injury. Importantly, in order to determine the molecular mechanism of Sevo in HIRI, the focus of the study was on microRNA (miR) regulation. By retrieving the microarray data in the Gene Expression Omnibus dataset (GSE72315), miR-218-5p was found to be significantly downregulated by Sevo. Moreover, miR-218-5p overexpression using agomiR-218-5p reversed the protective roles of Sevo against HIRI. Furthermore, GAB2, a positive regulator of PI3K/AKT signaling pathway, was found as a target gene of miR-218-5p. It was also found that the Sevo-mediated protective effects may be dependent on the activation of GAB2/PI3K/AKT. Collectively, these data revealed that Sevo alleviated HIRI in mice by restraining apoptosis, relieving oxidative stress and inflammatory response through the miR-218-5p/GAB2/PI3K/AKT pathway, which helps in understanding the novel mechanism of the hepatic-protection of Sevo.
\end{abstract}

Correspondence to: Professor Zhijun Cheng, Department of Anesthesiology, Xinhua Hospital, Chongming Branch, 25 Nanmen Road, Chongming, Shanghai 202150, P.R. China E-mail: zhijun_cheng@sohu.com

Key words: sevoflurane, hepatic ischemia/reperfusion injury, inflammatory response and apoptosis, microRNA-218-5p, GAB2/PI3K/AKT pathway

\section{Introduction}

Hepatic ischemia/reperfusion (I/R) injury (HIRI) is a pathophysiological condition post major liver surgeries or transplantation (1). Although the understanding on the HIRI mechanisms has advanced, no effective strategies have been developed. Considerable studies have demonstrated that inflammation and oxidative stress are the two most important mechanisms during the early stages of HIRI when Kupffer cells produce large amounts of reactive oxygen species (ROS) and pro-inflammatory factors, such as tumor necrosis factor (TNF)- $\alpha$, interleukin (IL)-1, and IL-6 (2-4). During later phases of I/R injury, these cytokines activate neutrophils, which further release cytokines, ROS, myeloperoxidase (MPO), and a variety of factors that exacerbate tissue damage. Therefore, it is important to identify effective strategies to mitigate inflammatory and oxidative stress to protect the liver from I/R injury.

Numerous studies have reported that volatile anesthetic can provide protective effects in various tissues and organ injury, including the liver (5-7). Sevoflurane (Sevo), a novel volatile anesthetic, is widely used in anesthetic practice. For example, Zhang et al (8) found that Sevo preconditioning exhibited a significant protective effect against cerebral I/R injury in a rat model. Ohsumi et al (9) showed that Sevo improved I/R injury through suppressing inflammation and apoptosis in a rat lung transplantation model. Liu et al (10) demonstrated that Sevo improved intestinal $\mathrm{I} / \mathrm{R}$ injury by inhibiting intestinal inflammatory reaction in rats. Zheng et al (11) reported that Sevo pretreatment has the beneficial effects on renal I/R injury through enhancing hypoxia-inducible factors- $2 \alpha$ expression in mice. Recently, several studies have indicated that Sevo can protect against HIRI through several mechanisms, including inhibition of apoptosis, production of ROS, and alleviating inflammatory responses $(12,13)$. More importantly, Sevo has been widely used during hepatobiliary surgery and was reported to improve clinical outcomes of liver resection with inflow occlusion, presumably due to hepatocyte protection from ischemic injury $(14,15)$. However, it remains unknown how Sevo regulates the inflammation and apoptosis in liver tissue against ischemia/reperfusion injury. 
MicroRNAs (miRNAs/miRs) are a group of endogenous, small non-coding RNAs that are of 19-22 nucleotides in length, which have been certified as essential regulators of gene expression at posttranscriptional levels $(16,17)$. An increasing body of evidence has supported that miRNAs are associated with pathological events during HIRI. For example, Xiao et al (18) showed that miR-219a-5p could attenuate HIRI-induced hepatic cells apoptosis via impairing TP53BP2 in mice. Xing et al (19) found that miR-27a-5p upregulation may alleviate the apoptosis of liver I/R injury in mice by targeting Bach1. Numerous studies have shown that Sevo exerts in vivo protection against I/R injury in different organs by regulating miRNAs (20-22). For example, Sevo ameliorated myocardial I/R injury in mice via upregulating miR-204 (23). Liao et al (24) showed that Sevo exerts protective effects on liver I/R injury by regulating NFKB3 expression via miR-9-5p. However, whether the positive effect of Sevo on HIRI is associated with miRNAs has not been fully explored.

The aim of the present study was to analyze the effects of Sevo on apoptosis, oxidative stress and inflammatory response in HIRI. Sevo treatment can suppress apoptosis, oxidative stress and inflammatory response induced by $\mathrm{I} / \mathrm{R}$ injury in mice. The hepatic-protective effect mechanism of Sevo may be associated with the miR-218-5p/GAB2/PI3K/AKT pathway. These findings may provide more evidence to clear the effect of Sevo on HIRI.

\section{Materials and methods}

Animals. Male BALB/c mice (age, 6-8 weeks; weight, 18-22 g) were obtained from the Shanghai SLAC Laboratory Animal Co. Ltd. All mice were housed at $22 \pm 1^{\circ} \mathrm{C}, 50 \pm 10 \%$ relative humidity, at 12-h light/dark cycles, and with free access to food and water. Animal procedures were approved by the Animal Care and Use Committee of the Shanghai Jiao Tong University School of Medicine (permit no. 2019-0033).

HIRI. All mice were fasted overnight before the ischemia/reperfusion operation. The animals were divided into three groups $(n=6)$ : Sham, HIRI, and Sevo + HIRI groups. All mice were anesthetized using an i.p. injection of sodium pentobarbital (40 mg/kg body weight; Sigma-Aldrich; Merck KGaA) and mice in the Sevo + HIRI group were then treated with $2 \%$ Sevo for the full duration of the surgical intervention. Liver ischemia was induced by clamping the portal vein, hepatic artery and bile duct of the left and median for $45 \mathrm{~min}$. At the end of the ischemia period, the vascular clamp was removed, and the liver was perfused for $2 \mathrm{~h}$. After 2-h reperfusion, $0.5 \mathrm{ml}$ cardiac blood was collected by sterile syringes without anticoagulant and centrifuged at $3,000 \mathrm{x} \mathrm{g}$ for $5 \mathrm{~min}$ at $4^{\circ} \mathrm{C}$ to separate the serum. Then, mice were euthanized by i.p. injection of pentobarbital sodium (40 mg/kg; Sigma-Aldrich; Merck KGaA), followed by cervical dislocation. Liver samples were excised and immediately snap frozen in liquid nitrogen or fixed in $10 \%$ formaldehyde for $24 \mathrm{~h}$ at $25^{\circ} \mathrm{C}$.

Therapy with agomiR-218-5p in mouse model. Agomir-218-5p (10 mg/kg) or agomiR-negative control (NC, $10 \mathrm{mg} / \mathrm{kg}$ ) (Guangzhou RiboBio Co., Ltd.) was injected into the mice using a glass micropipette (tip diameter, 20-40 $\mu \mathrm{m}$ ) by tail intravenous injection. Following injection $(24 \mathrm{~h})$, $45 \mathrm{~min}$ of ischemia and $2 \mathrm{~h}$ of reperfusion was performed. The agomiR-218-5p (5'-UUGUGCUUGAUCUAACCA UGU-3'), agomiR-NC (5'-UCACAACCUCCUAGAAAG AGUAGA-3'), antagomiR-218-5p (5'-AACACGAACUAG AUUGGUACA-3') and antagomiR-NC (5'-UUGUACUAC ACAAAAGUACUG-3') were synthesized by Shanghai GenePharma Co., Ltd.

Biochemical indicator analysis. Alanine aminotransferase (ALT; cat. no. C009-2-1), aspartate aminotransferase (AST; cat. no. C010-2-1), superoxide dismutase (SOD; cat. no. A001-1-1), malondialdehyde (MDA; cat. no. A003-1-2) levels and the activity of lactate dehydrogenase (cat. no. A020-2-2) were determined using commercial kits purchased from Jiancheng Biotechnology Co., Ltd. Serum cytokines/chemokines IL-1 $\beta$ (cat. no. 96-403), IL-6 (cat. no. 96-407), IFN- $\alpha$ (cat. no. 96-416), and IL-10 (cat. no. 96-408) were detected by enzyme-linked immunosorbent assay (ELISA) kits obtained from Merck KGaA.

Immunohistochemistry (IHC) staining. For IHC staining, liver tissues collected after ischemia/reperfusion injury in mice were fixed with $10 \%$ neutral formalin for $24 \mathrm{~h}$ at room temperature and then embedded in paraffin. Samples were sectioned at a thickness of $4 \mu \mathrm{m}$, and the sections were dewaxed and dehydrated. After antigen retrieval in citrate buffer at $95^{\circ} \mathrm{C}$ for $15 \mathrm{~min}$, the sections were blocked with $10 \%$ bovine serum albumin (cat. no. A1933; Sigma-Aldrich; Merck KGaA) overnight at $4^{\circ} \mathrm{C}$. Then, the sections were incubated with primary antibody against caspase-3 (cat. no. 9662; Cell Signaling Technology, Inc.; 1:100) at $4^{\circ} \mathrm{C}$ overnight. Subsequently, the sections were incubated with goat Anti-Rabbit IgG H\&L (Alexa Fluor ${ }^{\circledR} 488$; cat. no. ab150081; 1:2,000; Abcam) for $30 \mathrm{~min}$ at $37^{\circ} \mathrm{C}$. Finally, the immune-reactivity was visualized by staining with diaminobenzidine (DAB; cat. no. D-5637; Sigma-Aldrich; Merck $\mathrm{KGaA}$ ) at room temperature for 3 min and analyzed using a light microscope (Olympus BX microscope; magnification, x200).

miRNA microarray assay. Microarray dataset was obtained from theGeneExpressionOmnibus(GEO)database(https://www.ncbi. nlm.nih.gov/geo/query/acc.cgi?acc=GSE72315) and the GEO accession number is GSE72315. The GSE72315 dataset was based on Illumina HiSeq 2000 (Mus musculus) platform. GEO2R (www.ncbi.nlm.nih.gov/geo/geo2r/), an interactive web tool, was applied to compare the samples between two different groups under the same experimental condition. Differentially expressed miRNAs (DE-miRNAs) were then identified based on the fold change. The heat map of DE-miRNAs was created using a method of hierarchical clustering by GeneSpring GX, version 7.3 (Agilent Technologies, Inc.).

$R N A$ isolation and reverse transcription-quantitative (RT-q) $P C R$. Total RNA was extracted from liver tissues with a miRNeasy Mini kit (Qiagen $\mathrm{GmbH}$ ). RNA samples (10 ng) were subjected to reverse transcription to cDNA using Taqman MicroRNA Assays (Applied Biosystems; Thermo Fisher Scientific, Inc.) at $42^{\circ} \mathrm{C}$ for $1 \mathrm{~h}$. The U6 gene was used as a reference control for miR-218-5p. The RT-PCR reactions for the miRNAs was analyzed using SYBR ${ }^{\circledR}$ Premix Ex Taq ${ }^{\mathrm{TM}}$ 
(Takara Bio, Inc.) on an ABI Prism 7900 HT (Applied Biosystems; Thermo Fisher Scientific, Inc.). The primers used were as follows: miR-218-5p forward (F), 5'-CGAGTGCAT TTGTGCTTGATCTA-3'; reverse miR-218-5p (R), 5'-TAA TGGTCGAACGCCTAACGTC-3'; miR-219a-5p F, 5'-GCA GATGGCTCAGTTCAGCAG-3'; miR-219a-5p R, 5'-GTGCAG GGTCCGAGGT-3'; miR-93 F, 5'-TGCGGTTTGGCACTA GCACATT-3'; miR-93 R, 5'-CCAGTGCAGGGTCCGAGG T-3'; miR-17 F, 5'-GCCGCAAAGTGCTTACAGTG-3', miR-17 R, 5'-TGCAGGGTCCGAGGTAT-3'; miR-200c F, 5'-GGTAAT ACTGCCGGGTAAT-3'; miR-200c R, 5'-CAGTGCTGTCGT GAGT-3'; U6 F, 5'-GCTTCGGCAGCACATATACTAAAA T-3'; U6 R, 5'-CGCTTCAGAATTTGCGTGTCAT-3'. Relative expression levels were calculated using the $2^{-\Delta \Delta C q}$ method (25).

Luciferase reporter assay. The luciferase reporter vectors (pGL3-GAB2-3'UTR Wt and pGL3-GAB2-3'UTR Mut) were synthesized by GenePharma. 293 cells $\left(2 \times 10^{5} /\right.$ well) were seeded into 24-well plates and co-transfected with $0.2 \mu \mathrm{g}$ luciferase reporter vectors and $100 \mathrm{nM}$ agromiR-218-5p or antagromiR-218-5p using Lipofectamine ${ }^{\circledR} 2000$ (Invitrogen; Thermo Fisher Scientific, Inc.). After 48 h, luciferase activity was detected using the Dual-Luciferase Reporter Assay system (Promega Corporation). The relative firefly luciferase activities were measured by normalizing to Renilla luciferase activities.

Western blot analysis. Total cell protein was extracted using radio-immunoprecipitation assay buffer (Beyotime Institute of Biotechnology). The concentration of the protein was measured by the BCA protein assay kit (Beyotime Institute of Biotechnology). Protein samples $(40 \mu \mathrm{g})$ extracted from liver tissues were separated with 10\% SDS-PAGE and transferred onto PVDF (EMD Millipore) membranes. These membranes were then blocked with 5\% skim milk for $2 \mathrm{~h}$ at room temperature. Subsequently, membranes were incubated with primary antibodies against caspase-3 (cat. no. 9662), cleaved-caspase-3 (cat. no. 9664), GAB2 (cat. no. 3884), PI3K (p85) (cat. no. 17336), phosphorylated (p)-AKT (cat. no. 4060), AKT (cat. no. 4691), p-mTOR (cat. no. 5536), mTOR (cat. no. 2983) and $\beta$-actin (cat. no. 4970) (all antibodies were obtained from Cell Signaling Technology, Inc.; all dilutions were at 1:1,000) at $4^{\circ} \mathrm{C}$ overnight. Subsequently, the blots were washed with TBST and further incubated at room temperature with an anti-rabbit IgG, HRP-linked antibody (cat. no. 7074; Cell Signaling Technology, Inc.; 1:2,000) for $1 \mathrm{~h}$. The protein bands were visualized using ECL kit (GE Healthcare) and quantified with ImageJ (version 1.46; Rawak Software, Inc.).

Statistical analysis. Statistical analysis was performed using SPSS (version 13.0; SPSS, Inc.). Data were presented as means \pm SD. One-way ANOVA followed by Tukey's post-hoc tests was used to analyze the differences among multiple groups. $\mathrm{P}<0.05$ was considered to indicate a statistically significant difference. Each experiment was repeated at least three times.

\section{Results}

Protective effects of Sevo on HIRI. Sevo has been shown to exhibit protective effects in different tissue models of
I/R injury (26-29). In the present study, a mouse model of HIRI was used to evaluate the therapeutic effects of Sevo. As shown in Fig. 1A-C, the serum ALT and AST levels, as well as the activity of LDH, were significantly increased following I/R injury. Meanwhile, the liver enzyme levels were significantly decreased after Sevo pretreatment in mice; this indicates that Sevo improves liver function following I/R injury. Furthermore, the effects of Sevo on apoptosis were examined. Immunohistochemistry (IHC) staining showed that exposure of mice to $\mathrm{I} / \mathrm{R}$ leads to a significant increase in the caspase-3 expression levels. However, the elevated caspase-3 expression was significantly decreased after Sevo pretreatment (Fig. 1D). It was also found that the increased expression of cleaved caspase- 3 protein caused by HIRI was decreased by Sevo, as determined by western blot analysis (Fig. 1E). All data suggest that the Sevo pretreatment may attenuate HIRI through suppressing hepatocytes apoptosis in the mice.

Sevo treatment attenuates oxidative stress and inflammatory response. Excessive oxidative stress and excessive inflammatory response are well recognized as another contributory factor in the pathology of HIRI (30). Thus, the present study examined the protective effects of Sevo on oxidative stress. As shown in Fig. 2A and B, HIRI significantly increased the MDA levels, and markedly decreased the SOD levels compared with the Sham group. However, the MDA levels were significantly decreased, and SOD levels were markedly increased after Sevo pretreatment in mice. To further investigate the anti-inflammatory effects of Sevo, the expression levels of inflammatory factors (IL-1 $\beta$, IL-6, IL-10 and TNF- $\alpha$ ) were determined via ELISA in the liver tissue. It was shown that the TNF- $\alpha$, IL-1 $\beta$, IL-6 levels were significantly increased, and anti-inflammatory factor IL-10 levels were markedly decreased in HIRI group compared with the Sham group, whereas Sevo decreased the release of TNF- $\alpha$, IL-1 $\beta$, and IL- 6 and increased the levels of IL-10 in the liver tissue (Fig. 2C-F). The results suggest that Sevo may exert protective effect against HIRI through suppressing oxidative stress and inflammatory response.

Impact of Sevo on miRNAs during HIRI. It is reported that a number of miRNAs play important roles in Sevo protection in several animal models of I/R injury $(22,31)$. Thus, in order to explore the molecular mechanism of Sevo in HIRI, the present study focused on miRNA regulation. The DE-miRNAs were first analyzed via retrieving the microarray data in the GEO dataset (GSE72315). Custer analysis showed a significant difference in the expression of miRNAs between HIRI and Sham groups (Fig. 3A). Given published research has revealed the involvement of six of the DE-miRNAs, including miR-93, miR-133a-5p, miR-319-3p, miR-17, miR-200c and miR-218-5p, in HIRI (18,32-35), they were selected and their expression was validated in sham, HIRI, and HIRI + Sevo groups. Consistent with the aforementioned studies, miR-93, miR-133a-5p, miR-319-3p were significantly increased, while miR-17, miR-200c and miR-218-5p were dramatically decreased in HIRI group compared with Sham. Interestingly, the increased expression level of miR-218-5p in the HIRI group was notably decreased when pretreated with Sevo, whereas the expression levels of other five miRNAs were not altered following 
A

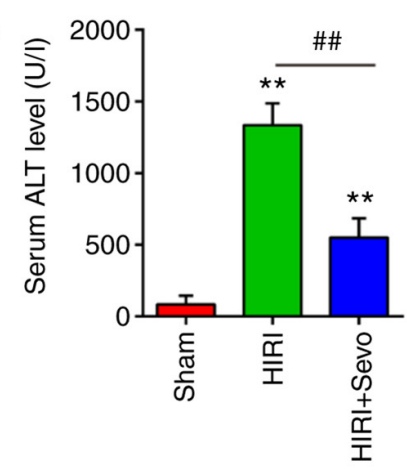

B

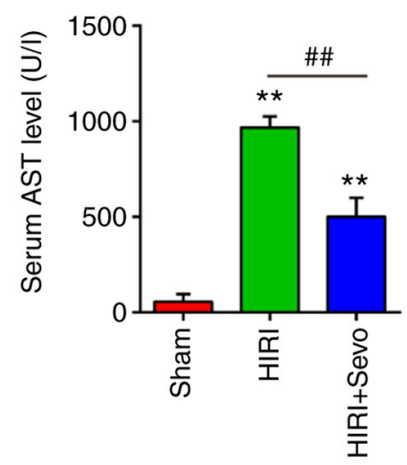

C

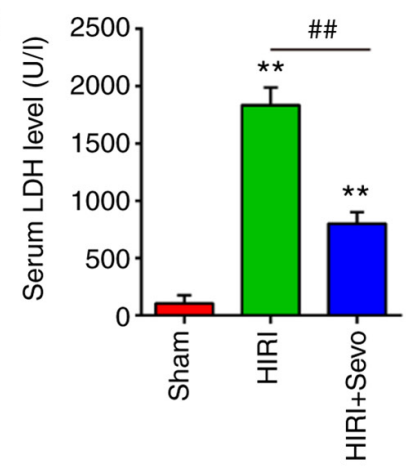

D

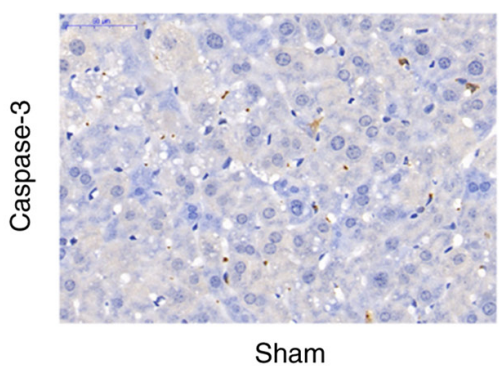

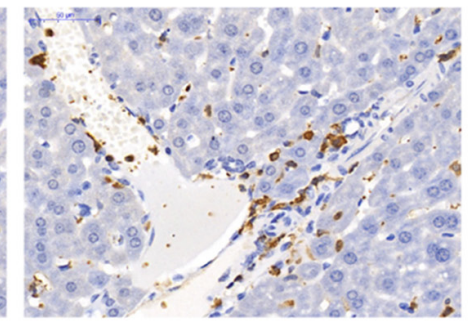

HIRI

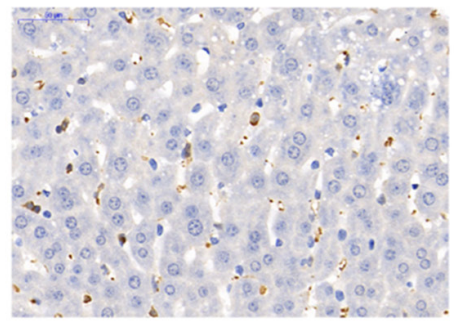

HIRI+Sevo
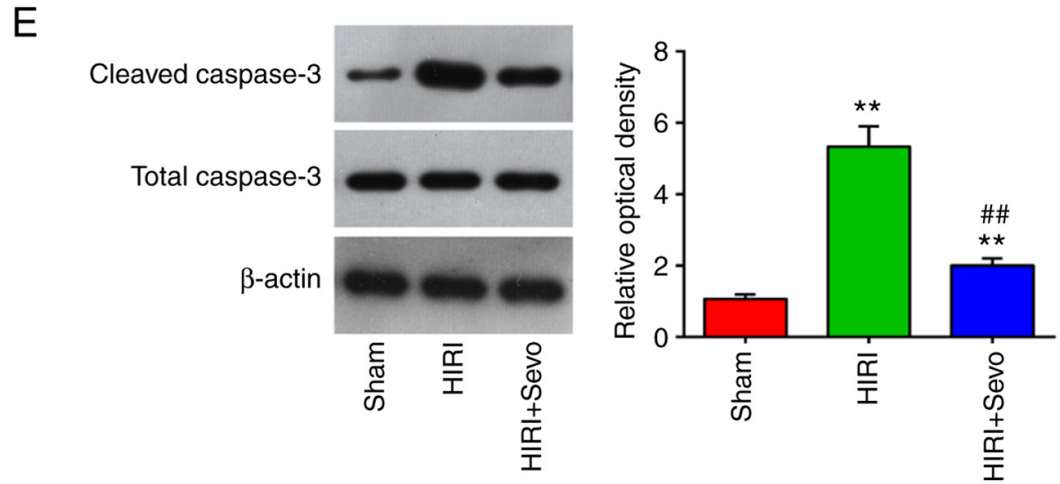

Figure 1. Sevo decreases HIRI. Mice were randomly divided into the sham, the HIRI and the HIRI + Sevo groups (n=6 mice/group). Animals in the I/R groups underwent $45 \mathrm{~min}$ ischemia and $2 \mathrm{~h}$ reperfusion and mice in the HIRI + Sevo were administered $2 \%$ sevo for the duration of the surgery. (A-C) Liver enzyme assays were performed in the three groups: Sham, HIRI and HIRI + Sevo groups ( $\mathrm{n}=6 /$ group). (D) The expression of caspase-3 was measured by immunohistochemistry. Magnification, x200. (E) The expression level of cleaved caspase-3 was measured by western blotting. Data are expressed as mean \pm standard deviation and analyzed by one-way analysis of variance. ${ }^{* *} \mathrm{P}<0.01$ vs. Sham group; ${ }^{\# \#} \mathrm{P}<0.01$ vs. HIRI group. HIRI, hepatic ischemia/reperfusion injury.

Sevo treatment (Fig. 3B). These data suggest that Sevo may exert protective effect against HIRI through regulation of miR-218-5p.

miR-218-5p mediated the protective effect of Sevo through the regulation of apoptosis. Since miR-218-5p was downregulated in response to Sevo treatment in mice model, it was speculated that it may play an important role in the protective effects of Sevo against HIRI. In order to test this, agomir-218-5p or agomiR-NC was injected into the mice model via tail intravenous injection. As show in Fig. 4A-C, Sevo inhibited the serum ALT and AST levels, and the activity of LDH in HIRI mice, whereas overexpression of miR-218-5p by agomiR-218-5p attenuated the inhibitory effect of Sevo on the serum ALT and AST levels, and the activity of LDH. Furthermore, apoptosis-associated proteins were evaluated using IHC in Sevo-treated HIRI mice, following agomir-miR-129-5p injection. IHC staining showed that Sevo treatment suppressed the expression of caspase-3 in HIRI mice, while these effects of Sevo were reversed by agomiR-218-5p (Fig. 4D). The similar effects were also observed in the expression levels of cleaved caspase-3, determined by western blot analysis (Fig. 4E). Cumulatively, these results suggested that miR-218-5p mediated the protective effect of Sevo against HIRI in mice through the regulation of apoptosis.

miR-218-5p mediates the protective effect of Sevo through the regulation of oxidative stress and inflammatory response. It was further evaluated whether the inhibitory effects of Sevo on oxidative stress were mediated by miR-218-5p, by measuring the MDA and SOD levels. As shown in Fig. 5A and B, Sevo decreased MDA levels, and increased SOD levels in HIRI mice, whereas the inhibitory effect of Sevo on oxidative stress was attenuated by agomiR-218-5p. Furthermore, inflammatory cytokine production was evaluated using ELISA in Sevo-treated HIRI mice following agomir-miR-129-5p injection. It was found that Sevo suppressed the levels of 
A

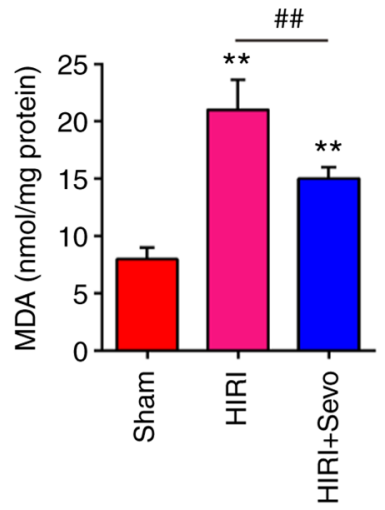

D

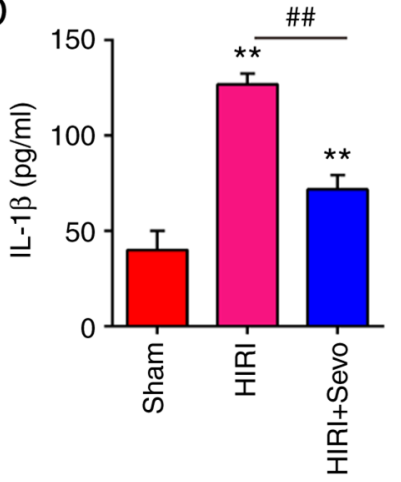

B

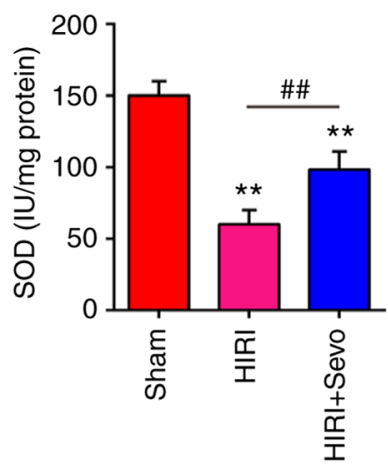

E

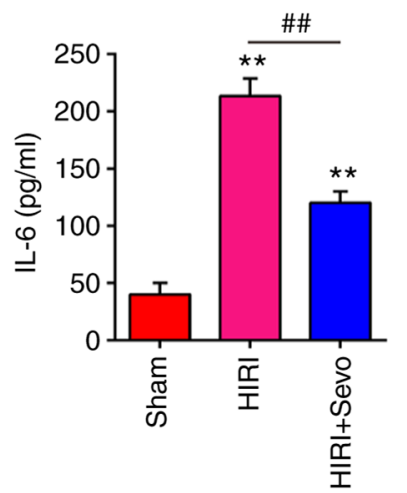

C

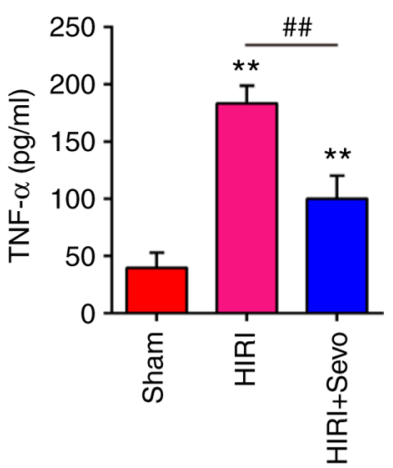

F

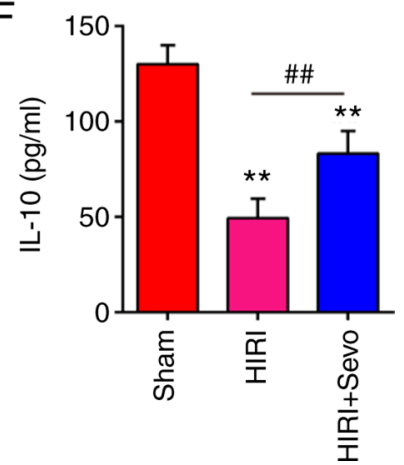

Figure 2. Sevo attenuates oxidant stress and inflammatory response. Mice were randomly divided into the sham, the HIRI and the HIRI + Sevo groups ( $\mathrm{n}=6 \mathrm{rats} / \mathrm{group}$ ). Animals in the ischemia/reperfusion groups underwent $45 \mathrm{~min}$ ischemia and $2 \mathrm{~h}$ reperfusion, and mice in the HIRI + Sevo were administered $2 \%$ sevo for the duration of the surgery. (A and B) The contents of MDA and SOD were measured by ELISA assays. (C-F) The inflammatory cytokines including TNF- $\alpha$, IL-1 $\beta$, IL- 6 and IL-10, were evaluated by ELISA assays. Data are expressed as mean \pm standard deviation and analyzed by one-way analysis of variance. ${ }^{* *} \mathrm{P}<0.01$ vs. Sham group; ${ }^{\# \#} \mathrm{P}<0.01$ vs. HIRI group. Sevo, sevoflurane; ELISA, enzyme-linked immunosorbent assay; HIRI, hepatic ischemia/reperfusion injury; MDA, malondialdehyde; SOD, superoxide dismutase; TNF, tumor necrosis factor; IL, interleukin.

TNF- $\alpha$, IL-1 $\beta$ and IL- 6 , and increased IL-10 levels in HIRI mice, while the inhibitory effect of Sevo on inflammatory response were significantly reversed by agomiR-218-5p (Fig. 5C-F). These results suggested that miR-218-5p mediated the protective effect of Sevo through the regulation of oxidative stress and inflammatory response.

GAB2 is a direct target of $m i R-218-5 p$. To further investigate the mechanisms by which miR-218-5p mediated the protective effect of Sevo against HIRI, the potential target genes of miR-218-5p were determined using PicTar version 2007 and TargetScan Release 7.0. As presented in Fig. 6A, miR-218-5p contained a sequence complementary to GAB2. Subsequently, the transfection efficiency of agomiR-218-5p and antagomir-218-5p was assessed by RT-qPCR. As shown in Fig. 6B, agomiR-218-5p significantly increased the miR-218-5p levels, while antagomir-218-5p markedly decreased the miR-218-5p levels. Previous studies have reported that GAB2 is a target of miR-218-5p in prostate cancer ( $\mathrm{PCa}$ ) (36). To further validate these findings, a luciferase assay was performed. As expected, agomiR-218-5p effectively attenuated luciferase activity of GAB2-3'UTR wt, while antagomiR-218-5p promoted the luciferase activity of GAB2-3'UTR wt. However, either agomiR-218-5p or antagomiR-218-5p had no effect on the luciferase activity of GAB2-3'UTR mut (Fig. 6C). Subsequently, the influence of miR-218-5p on GAB2 was measured at the mRNA level by
RT-qPCR analysis. As shown in Fig. 6D, miR-218-5p overexpression significantly downregulated the expression of GAB2, while miR-218-5p knockdown upregulated its expression. Taken together, these data indicate that miR-218/GAB2 axis may play an important role in the protective effect of Sevo against HIRI.

Sevo activated the PI3K/AKT pathway through miR-218/GAB2 axis. GAB2 has been shown to activate the PI3K/AKT pathway (37), and PI3K/AKT has a key role in HIRI $(38,39)$. To investigate whether Sevo influences the PI3K/AKT activity, the expression levels of downstream proteins in the PI3K/AKT signaling pathway, namely PI3K (p85), p-AKT, p-mTOR and mTOR, were evaluated in Sevo-treated HIRI mice following agomir-miR-129-5p injection. The results showed that Sevo significantly increased the GAB2, PI3K (p85), p-AKT and p-mTOR expression levels in HIRI mice, while these promoting effects were reversed by agomiR-218-5p (Fig. 7A and B). Overall, these results demonstrate that Sevo promoted the GAB2-mediated activation of PI3K/AKT pathway through suppressing miR-218-5p.

\section{Discussion}

The present study findings revealed that Sevo improved HIRI via its antioxidant, anti-inflammatory, and anti-apoptotic activities in mice. Notably, the data indicated that 
A

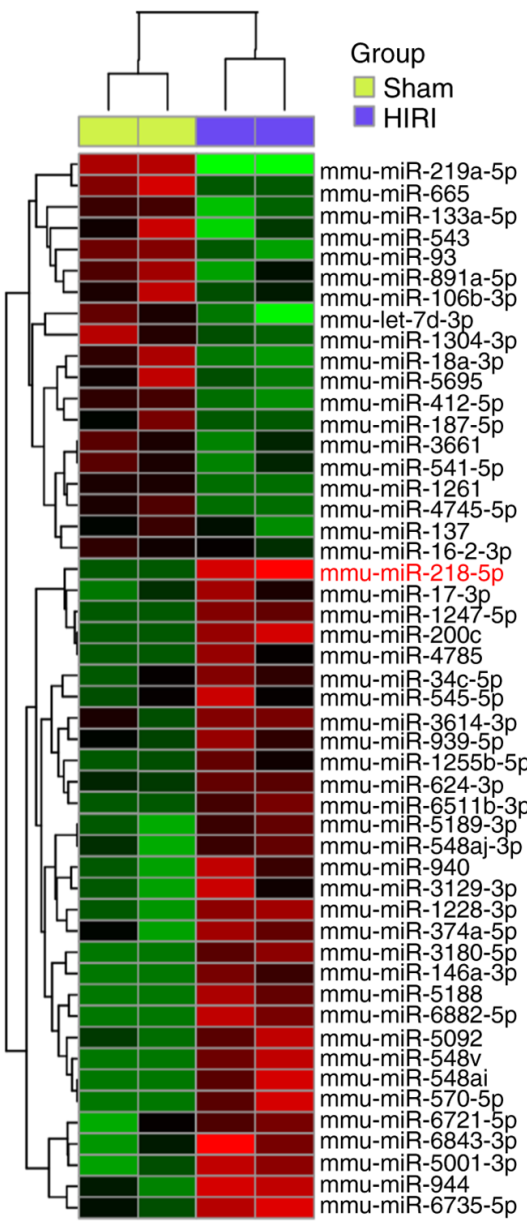

B

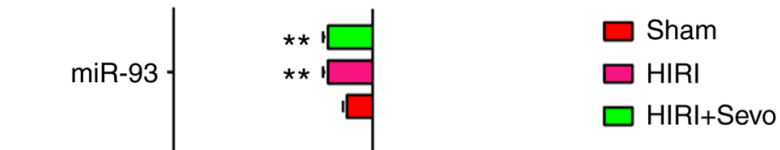

Figure 3. Sevo upregulates the expression level of miR-218-5p. (A) Heat map analysis of the miRNA expression levels of HIRI and Sham groups were created using a method of hierarchical clustering by GeneSpring GX, version 7.3. Rows, groups; columns, miRNAs. Color key indicates miRNA expression value: Red, highest; green, lowest. Microarray data obtained from Gene Expression Omnibus (GSE72315). (B) The expression levels of miR-93, miR-133a-5p, miR-219a-5p, miR-200c, miR-17 and miR-218-5p were detected in Sham, HIRI, and HIRI + Sevo groups by reverse transcription-quantitative PCR. All data are expressed as mean \pm standard deviation and analyzed by one-way analysis of variance. ${ }^{*} \mathrm{P}<0.05,{ }^{* *} \mathrm{P}<0.01$ vs. Sham group; ${ }^{\# \#} \mathrm{P}<0.01$ vs. HIRI group. Sevo, sevoflurane; miRNA/miR, microRNA; HIRI, hepatic ischemia/reperfusion injury.

Sevo exerts its protective role against HIRI through miR-218-5p/GAB2/PI3K/AKT axis (Fig. 8). The present study provides a novel mechanism for understanding the pharmacological effects of Sevo on HIRI.

A growing number of studies have suggested that apoptosis, oxidative stress and inflammatory response are prominent features of HIRI. Among these, oxidative stress refers to the elevated production of intracellular ROS, which can induce protein dysfunction, DNA damage and lipid peroxidation, resulting in the apoptosis and death of hepatocytes, playing a pivotal role in the process of HIRI. In this regard, different interventions (pharmacological and nutritional) can attenuate oxidative stress and/or increase the antioxidant response to prevent liver ischemia/reperfusion injury. For example, N-3 polyunsaturatedfatty acids (n-3PUFA) alleviated liverIR-injury through the suppression of oxidative stress in rats (40). Another study also demonstrated that thyroid hormone $\left(\mathrm{T}_{3}\right)$ administration exerts significant protection against liver I/R injury through decreasing liver oxidative stress in the rats (41). Sevo as a volatile anesthetic, has been reported to exhibit protective effect against various $I / R$ injury including liver $I / R$ injury, due to its anti-apoptotic, antioxidant and anti-inflammatory effects $(42,43)$. For example, Yu et al (44) found that Sevo protected rat hearts against cardiac I/R injury via ameliorating mitochondrial impairment and oxidative stress. In the case of liver, Mikrou et al (45) indicated that Sevo preconditioning prevent rat livers from I/R injury through suppressing inflammatory response and oxidative stress. The results of the present study revealed that Sevo markedly suppressed the apoptosis, oxidative stress and inflammatory response in mice following $\mathrm{I} / \mathrm{R}$ injury, which is in accordance with the findings of studies by Liao et al (24). These results suggested that Sevo exerts protective effects against liver $\mathrm{I} / \mathrm{R}$ injury in mice. Although a number of studies have attempted to investigate the possible mechanisms underlying the hepatic-protective effects of Sevo, the complete mechanisms have not yet been elucidated.

Growing evidence demonstrated that several miRNAs play key role in various types of $I / R$ injury, while the protective effects of Sevo that operate through targeting miRNAs have also been widely reported. Huang et al (46) found that miR-155 is involved in Sevo-mediated cardioprotection against myocardial I/R injury. Tan et al (23) showed that Sevo could upregulate miR-204 to ameliorate myocardial ischemia/reperfusion (I/R) injury in mice by inhibiting Cotl1 expression. In the present study, a large set of miRNAs were found to be significantly deregulated during HIRI and miR-218-5p was the most significantly 
A

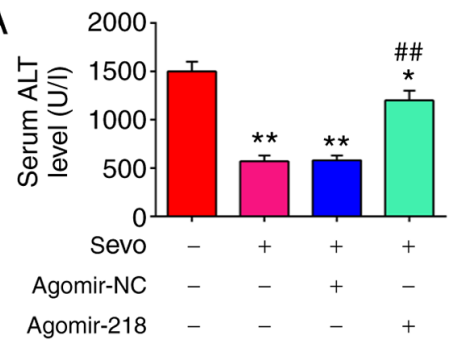

B

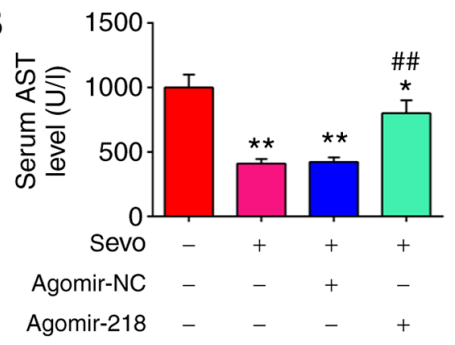

C

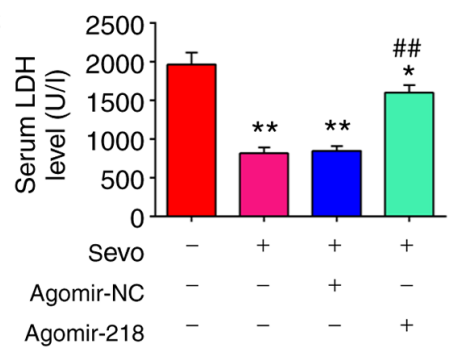

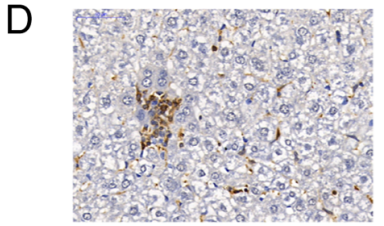

HIRI

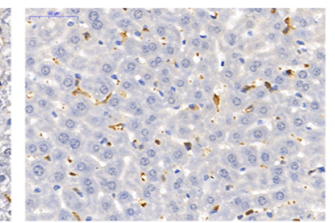

HIRI+Sevo

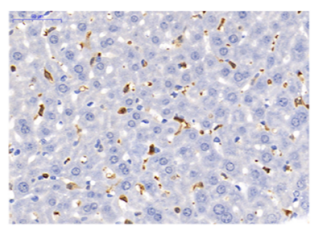

HIRI+Sevo+agomir-NC

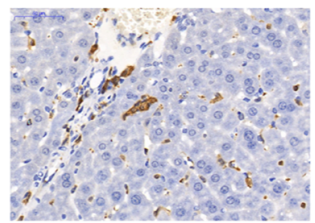

HIRI+Sevo+agomir-28

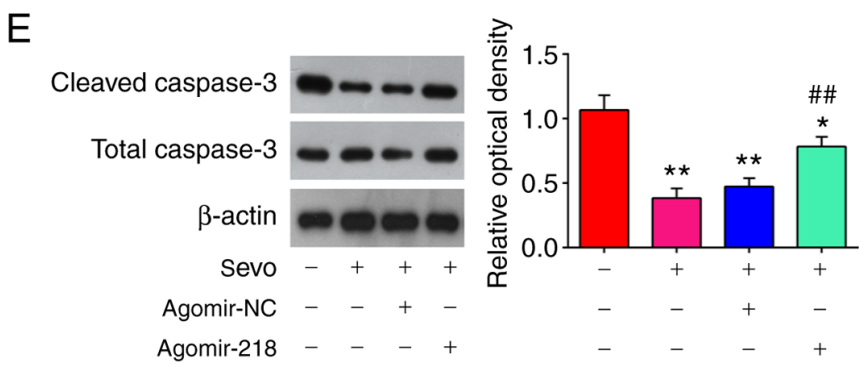

Figure 4. Sevo decreases HIRI through the regulation of miR-218-5p. The agomiR-218-5p and agomiR-NC were injected into mice, and the mice were subjected to I/R, post-treatment with inhalation of $2 \%$ Sevo. (A-C) Liver enzyme assays were performed in the four groups: HIRI, HIRI + Sevo, HIRI + Sevo + agomiR-218-5p, and HIRI + Sevo + agomiR-NC groups (n=6/group). (D) The expression level of caspase-3 was measured by immunohistochemistry. Magnification, x200. (E) The expression level of cleaved caspase-3 was measured by western blotting. Data are expressed as mean \pm standard deviation and analyzed by one-way analysis of variance. ${ }^{\mathrm{P}} \mathrm{P}<0.05,{ }^{* *} \mathrm{P}<0.01$ vs. HIRI group; ${ }^{\# *} \mathrm{P}<0.01$ vs. HIRI + Sevo group. Sevo, sevoflurane; miRNA/miR, microRNA; $\mathrm{NC}$, negative control; HIRI, hepatic ischemia/reperfusion injury; ALT, alanine aminotransferase; AST, aspartate aminotransferase; LDH, lactate dehydrogenase.

A
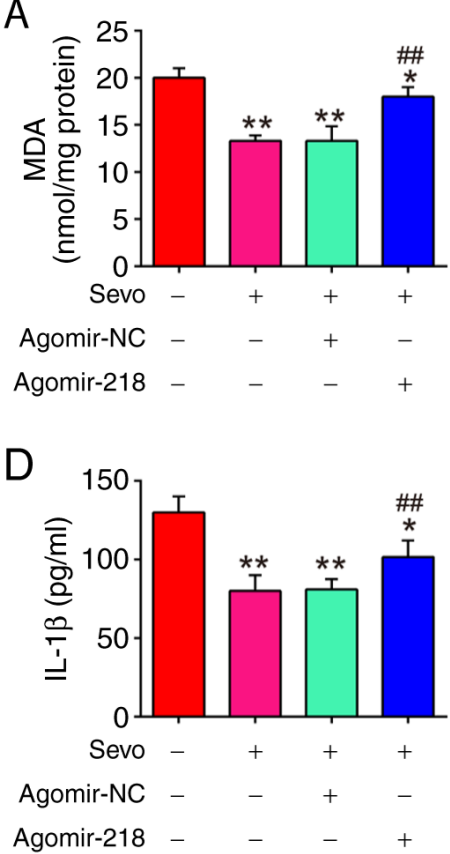

$\mathrm{B}$

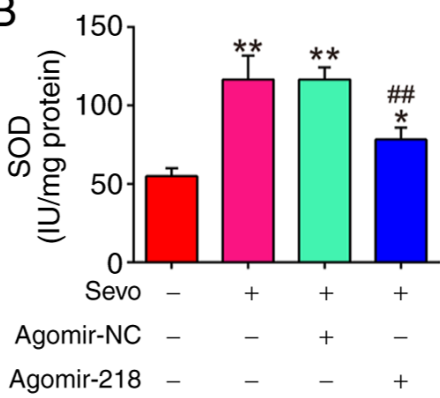

$E$

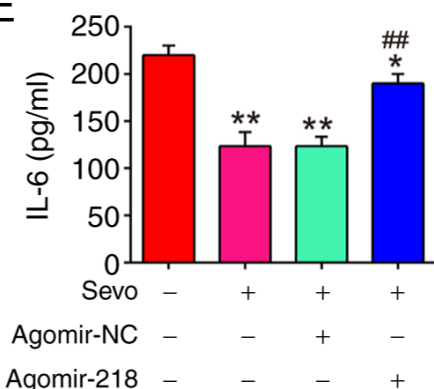

C

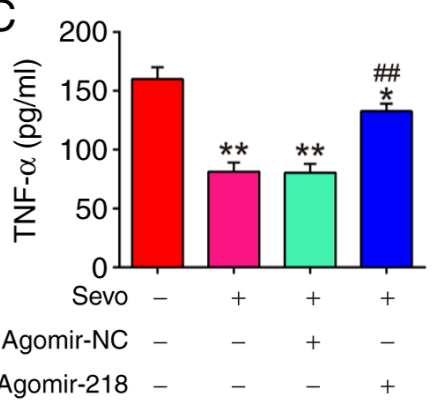

$\mathrm{F}$

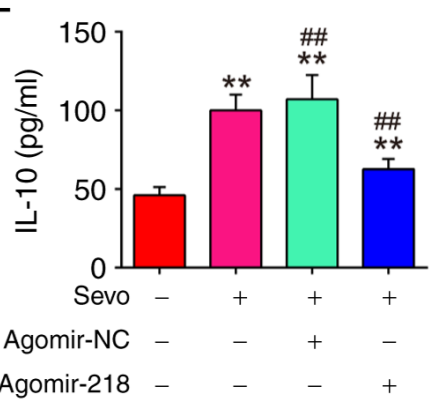

Figure 5. Sevo attenuates oxidant stress and inflammatory response through the regulation of miR-218-5p. The agomiR-218-5p and agomiR-NC were injected into mice. Then, the mice were subjected to ischemia/reperfusion injury and post-treatment with inhalation of $2 \%$ Sevo. (A and B) The contents of MDA and SOD were measured by ELISA assays. (C-F) The inflammatory cytokines, including TNF- $\alpha$, IL-1 $\beta$, IL-6 and IL-10, were evaluated by ELISA assays. Data are expressed as mean \pm standard deviation and analyzed by one-way analysis of variance. ${ }^{*} \mathrm{P}<0.05,{ }^{* * *} \mathrm{P}<0.01$ vs. HIRI group; ${ }^{\# \#} \mathrm{P}<0.01$ vs. HIRI + Sevo group. Sevo, Sevoflurane; miR, microRNA; NC, negative control; ELISA, enzyme-linked immunosorbent assay; HIRI, hepatic ischemia/reperfusion injury; MDA, malondialdehyde; SOD, superoxide dismutase; TNF, tumor necrosis factor; IL, interleukin. 


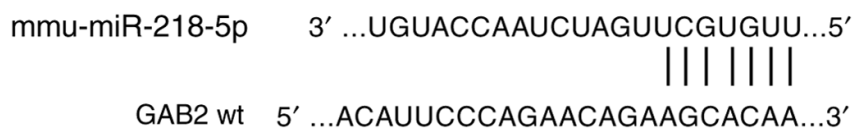

GAB2 mut 5' ...ACAUUCCCAGAACAGACAUGAGC...3'
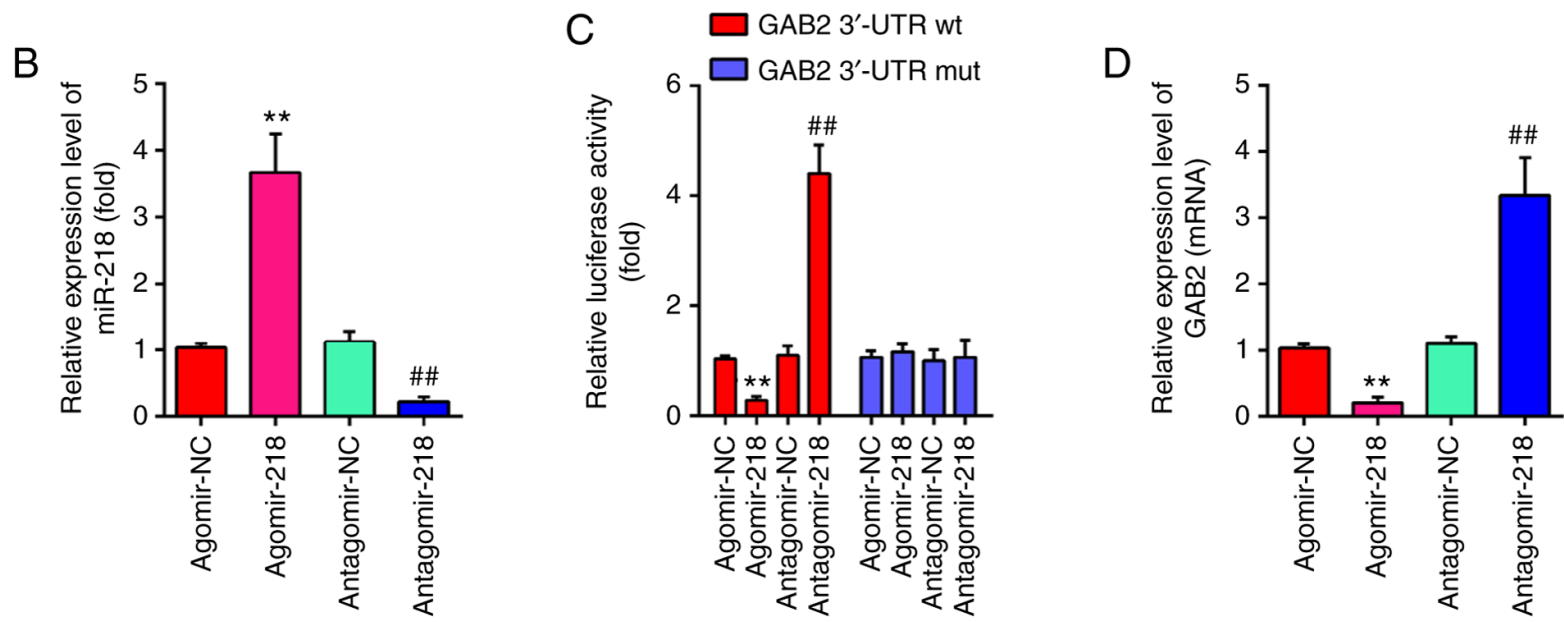

Figure 6. GAB2 is a direct target of miR-218-5p. (A) miR-218-5p-binding sequences in the 3'-UTR of GAB2 and mutated sites in 3'-UTR of GAB2. (B) The transfection efficiency of agomiR-218-5p and antagomir-218-5p was evaluated by RT-qPCR. (C) AgomiR-218-5p suppressed the luciferase activity of constructs containing the 3'-UTR segment of GAB2, while antagomiR-218-5p significantly increased the luciferase activity of constructs containing the 3'-UTR segment of GAB2 (n=3). (D) The mRNA expression level of GAB2 was detected by RT-qPCR after treatment with agomiR-218-5p or antagomiR-218-5p (n=3). All data are expressed as mean \pm standard deviation and analyzed by one-way analysis of variance. ${ }^{* * *} \mathrm{P}<0.01$ vs. agomiR-NC group; ${ }^{\# \#} \mathrm{P}<0.01$ vs. antagomiR-NC group. miR, microRNA; NC, negative control; UTR, untranslated region; RT-qPCR, reverse transcription-quantitative PCR; wt, wild type; mut, mutant.
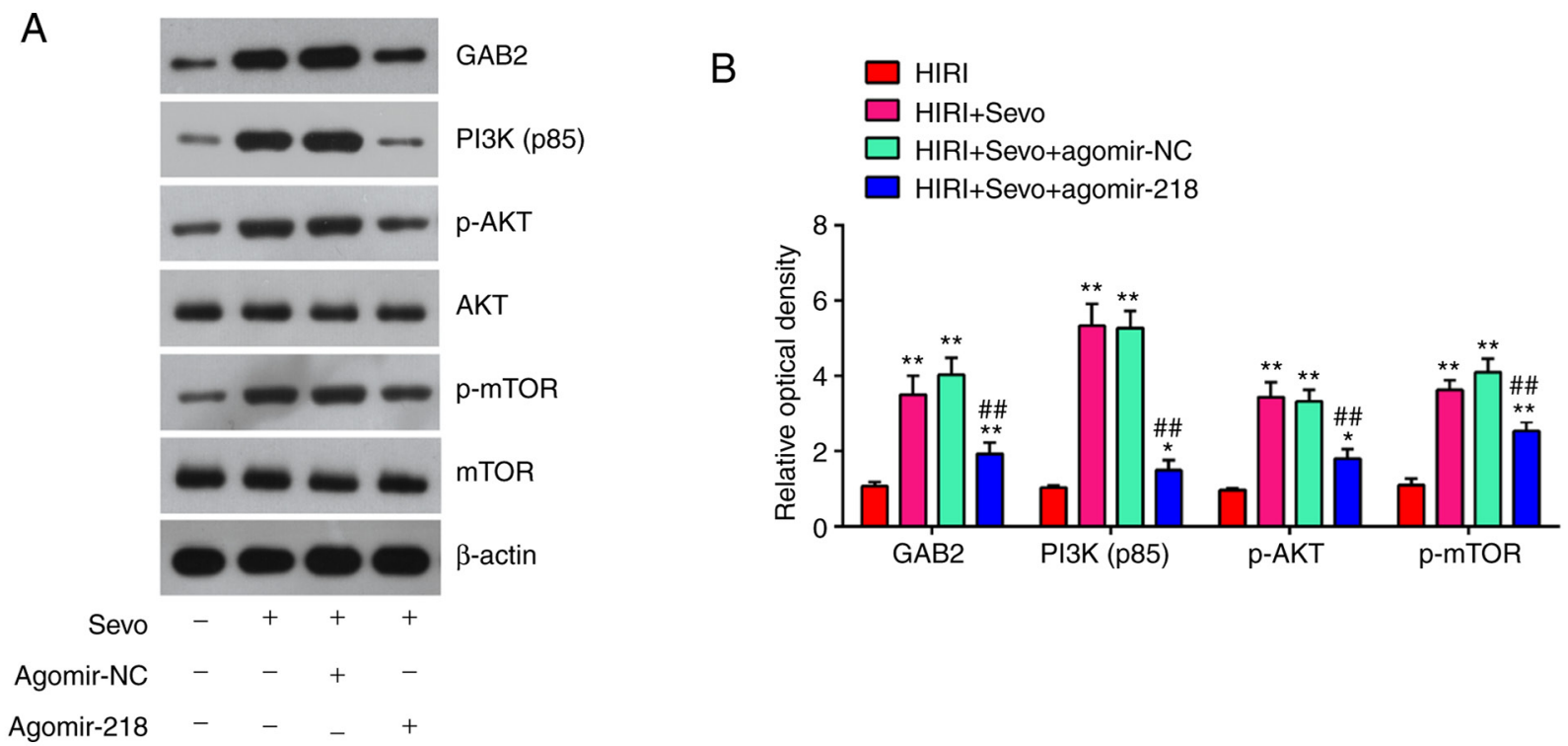

Figure 7. Sevo blocks the activation of GAB2/PI3K/AKT signaling pathway via decreasing miR-218. The agomiR-218-5p and agomiR-NC were injected into mice, and then, mice were subjected to ischemia/reperfusion and post-treatment with inhalation of $2 \%$ Sevo. (A) Protein expression levels of GAB2, PI3K (p85), p-AKT, AKT, p-mTOR and mTOR were detected by western blotting. (B) The bands were semi-quantitatively analyzed by using Image J software and normalized to $\beta$-actin density. All data are expressed as mean \pm standard deviation and analyzed by one-way analysis of variance. ${ }^{*} \mathrm{P}<0.05$, ${ }^{* *} \mathrm{P}<0.01 \mathrm{vs}$. HIRI group; ${ }^{\# \#} \mathrm{P}<0.01$ vs. HIRI + Sevo + agomir-NC group. Sevo, Sevoflurane; miR, microRNA; NC, negative control; p-, phosphorylated-.

downregulated miRNA by Sevo. Previous studies have demonstrated that miR-218-5p displayed the protective effect in various organs against injury, including I/R injury. For example, Zhu et al (47) showed that miR-218-5p inhibition protected against oxygen-glucose deprivation/reperfusion-induced injuries in PC12 cells through decreasing inflammatory cytokines secretion, oxidative stress status and apoptosis rate. Su et al (48) found that miR-218 downregulation could alleviate 


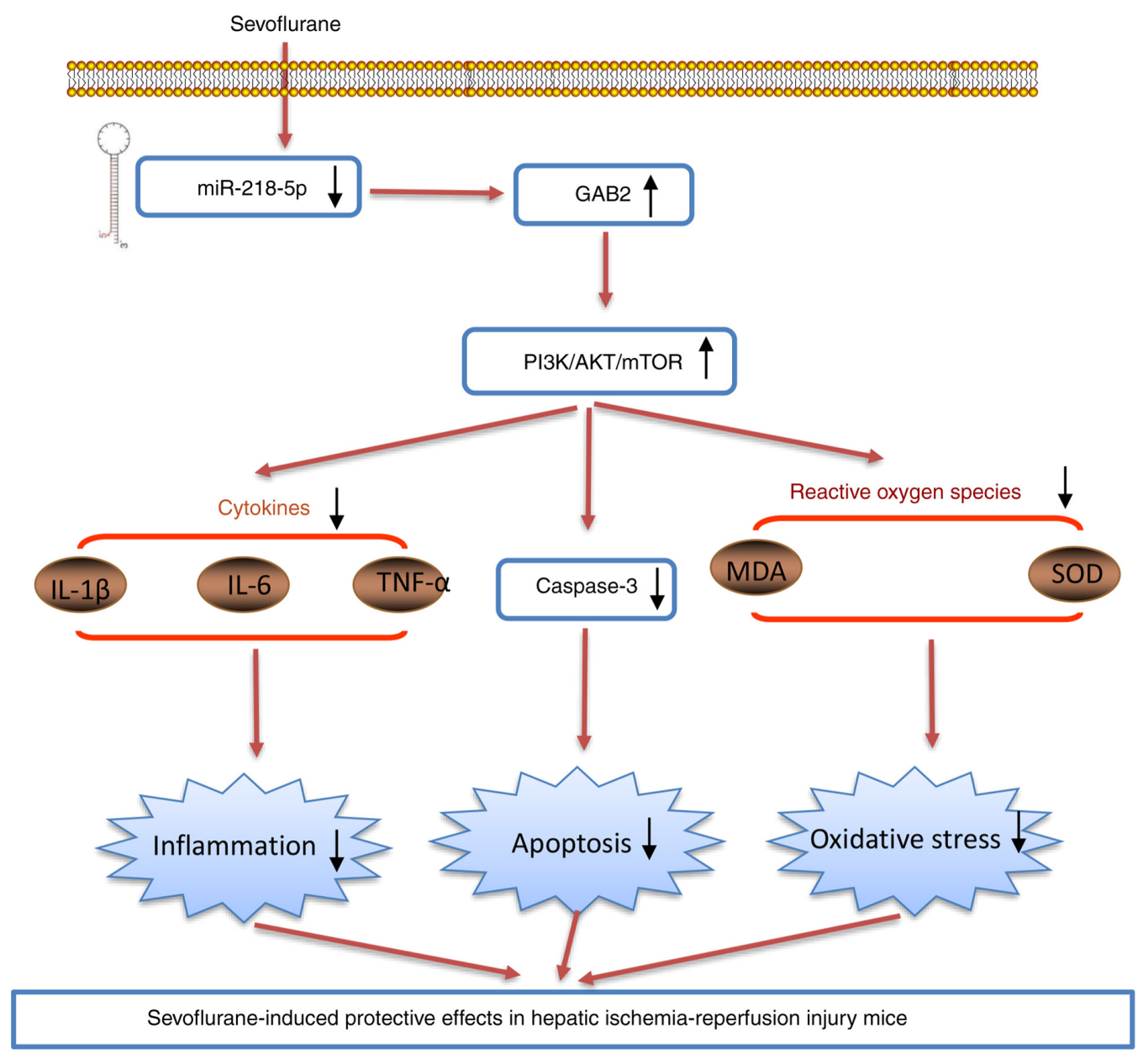

Figure 8. Sevoflurane downregulates the expression levels of miR-218-5p, which promotes GAB2 protein expression, subsequently activating the PI3K/AKT pathway. This finally results in the decrease in apoptosis, oxidative stress and inflammatory response, thus alleviating liver ischemia/reperfusion injury. miR, microRNA; MDA, malondialdehyde; SOD, superoxide dismutase; TNF, tumor necrosis factor; IL, interleukin.

high-glucose-induced renal proximal tubule injury by targeting GPRC5A. Li and Zhao (49) demonstrated that downregulation of miR-218 relieved neuropathic pain by regulating suppressor of cytokine signaling 3 in a rat model of chronic constriction injury. A recent study displayed that miR-218-5p was found to be significantly upregulated in drug-induced acute liver injury in mice (50). Thus, it was hypothesized that miR-218-5p was involved in the protective effect of Sevo on HIRI. The present study demonstrated that miR-218-5p overexpression reversed the anti-apoptotic, anti-oxidant and anti-inflammatory effects of Sevo on HIRI in mice. All these data suggest that miR-218-5p is involved in the protective role of Sevo against HIRI. However, the precise molecular mechanism needs further research to be understood in more depth.

PI3K and its downstream protein AKT have been regarded as a potential therapeutic target in HIRI $(51,52)$ which was positively regulated by GAB2, a member of the GRB2-associated binder (Gab) family (53). Li et al (54) showed that docosahexaenoic acid (DHA) ameliorated I/R-induced injury by inhibiting pyroptosis of hepatocytes through the PI3K/Akt pathway. Another study showed that high concentrations of hydrogen $(\mathrm{HCH})$ inhalation protect the liver against $\mathrm{I} / \mathrm{R}$ injury through the $\mathrm{A}_{2 \mathrm{~A}}$-dependent PI3K/Akt pathway in a mouse HIRI model (55). In the present study, GAB2 was identified as a direct target of miR-218-5p, which is consisted with a previous report (36). It is well reported that miR-218 inhibited the tumor angiogenesis in human cancer via regulating the GAB2/PI3K/AKT signaling pathway in various cancer types $(36,56)$. Thus, it was speculated that Sevo may have protective effects on HIRI via modulating the GAB2/AKT/mTOR signaling pathway by increasing miR-218-5p expression. As expected, Sevo treatment could inhibit the GAB2/AKT/mTOR signaling pathway in mice following I/R injury, but it was reactivated by miR-218-5p upregulation. Collectively, all these data suggest that Sevo may improve HIRI through the miR-218-5p/GAB2/PI3K/Akt pathway.

Accumulating evidence indicates that mitochondrial dysfunction plays a key role in the physiopathology of HIRI (57). It has been reported that mitochondrial dysfunction may lead to ATP production capacity to decrease or disorder, which further results in excessive production of ROS, finally causing structural damage to mitochondria and even cell death, a hall marker of I/R injury (58). Ortiz et al (59) reported that the co-administration of the lipid metabolism modulator DHA and the antioxidant hydroxytyrosol prevented the development of liver steatosis through the suppression of mitochondrial dysfunction induced by high-fat diet. Okatani et al (60) showed that edaravone, a potent free radical scavenger, protected against mitochondrial injury, 
which prevents mitochondrial oxidative stress and improves ischemia/reperfusion-induced hepatic energy metabolism. In addition, Mukhopadhyay et al (61) demonstrated that mitochondrial antioxidants, such as Mito-Q and Mito-CP, could suppress HIR-induced apoptotic cell death and liver dysfunction by decreasing mitochondrial damage. Therefore, the potential effect of Sevo on the prevention of mitochondrial dysfunction in $\mathrm{I} / \mathrm{R}$ injury will be studied in the future.

In conclusion, the present study findings indicated that Sevo can ameliorate liver I/R injury, which is at least partially associated with the miR-218-5p/GAB2-mediated PI3K-Akt pathway activation. These results represent an in-depth study of the molecular mechanism of Sevo in the treatment of HIRI and offer strong proof for the clinical value of this volatile anesthetic. Sevo and miRNAs may also provide a potential therapeutic strategy for the clinical treatment of hepatic disease.

\section{Acknowledgements}

Not applicable.

\section{Funding}

No funding was received.

\section{Availability of data and materials}

All data generated or analyzed during this study are included in this published article.

\section{Authors' contributions}

HJ, HL and HZ performed all the experiments and collected the data. $\mathrm{HJ}$ and $\mathrm{ZC}$ conceived and designed the study. $\mathrm{HJ}$ and $\mathrm{ZC}$ wrote the main manuscript and analyzed the data. $\mathrm{HJ}$ and $\mathrm{ZC}$ confirm the authenticity of all the raw data. All authors read and approved the final version of the manuscript.

\section{Ethics approval and consent to participate}

Animal procedures were approved by the Animal Care and Use Committee the Shanghai Jiao Tong University School of Medicine (permit no. 2019-0033).

\section{Patient consent for publication}

Not applicable.

\section{Competing interests}

The authors declare that they have no competing interests.

\section{References}

1. Jaeschke H: Molecular mechanisms of hepatic ischemiareperfusion injury and preconditioning. Am J Physiol Gastrointest Liver Physiol 284: G15-G26, 2003.

2. Jaeschke H and Woolbright BL: Current strategies to minimize hepatic ischemia-reperfusion injury by targeting reactive oxygen species. Transplant Rev (Orlando) 26: 103-114, 2012.
3. Li XJ and Ye QF: Fucoidan reduces inflammatory response in a rat model of hepatic ischemia-reperfusion injury. Can J Physiol Pharmacol 93: 999-1005, 2015.

4. Datta G, Fuller BJ and Davidson BR: Molecular mechanisms of liver ischemia reperfusion injury: Insights from transgenic knockout models. World J Gastroenterol 19: 1683-1698, 2013.

5. Kersten JR, Schmeling TJ, Hettrick DA, Pagel PS, Gross GJ and Warltier DC: Mechanism of myocardial protection by isoflurane. Role of adenosine triphosphate-regulated potassium (KATP) channels. Anesthesiology 85: 794-807; discussion 27A, 1996.

6. Schlack W, Preckel B, Barthel H, Obal D and Thamer V: Halothane reduces reperfusion injury after regional ischaemia in the rabbit heart in vivo. Br J Anaesth 79: 88-96, 1997.

7. Jeong JS, Kim D, Kim KY, Ryu S, Han S, Shin BS, Kim GS, Gwak MS and Ko JS: Ischemic preconditioning produces comparable protection against hepatic ischemia/reperfusion injury under isoflurane and sevoflurane anesthesia in rats. Transplant Proc 49: 2188-2193, 2017.

8. Zhang Y, Shan Z, Zhao Y and Ai Y: Sevoflurane prevents miR-181a-induced cerebral ischemia/reperfusion injury. Chem Biol Interact 308: 332-338, 2019.

9. Ohsumi A, Marseu K, Slinger P, McRae K, Kim H, Guan Z, Hwang DM, Liu M, Keshavjee S and Cypel M: Sevoflurane attenuates ischemia-reperfusion injury in a rat lung transplantation model. Ann Thorac Surg 103: 1578-1586, 2017.

10. Liu C, Ding R, Huang W, Miao L, Li J and Li Y: Sevoflurane protects against intestinal ischemia-reperfusion injury by activating peroxisome proliferator-activated receptor gamma/nuclear factor- $\mathrm{B}$ pathway in rats. Pharmacology 105: 231-242, 2020.

11. Zheng B, Zhan Q, Chen J, Xu H and He Z: Sevoflurane pretreatment enhance HIF-2 $\alpha$ expression in mice after renal ischemia/reperfusion injury. Int J Clin Exp Pathol 8: 13114-13119, 2015.

12. Bedirli N, Ofluoglu E, Kerem M, Utebey G, Alper M, Yilmazer D, Bedirli A, Ozlu O and Pasaoglu H: Hepatic energy metabolism and the differential protective effects of sevoflurane and isoflurane anesthesia in a rat hepatic ischemia-reperfusion injury model. Anesth Analg 106: 830-837, 2008.

13. Li J, Yuan T, Zhao X, Lv GY and Liu HQ: Protective effects of sevoflurane in hepatic ischemia-reperfusion injury. Int J Immunopathol Pharmacol 29: 300-307, 2016.

14. Shiraishi S, Cho S, Akiyama D, Ichinomiya T, Shibata I, Yoshitomi O, Maekawa T, Ozawa E, Miyaaki H and Hara T: Sevoflurane has postconditioning as well as preconditioning properties against hepatic warm ischemia-reperfusion injury in rats. J Anesth 33: 390-398, 2019.

15. Beck-Schimmer B, Roth Z'graggen B, Booy C, Köppel S, Spahn DR, Schläpfer M and Schadde E: Sevoflurane protects hepatocytes from ischemic injury by reducing reactive oxygen species signaling of hepatic stellate cells: Translational findings based on a clinical trial. Anesth Analg 127: 1058-1065, 2018.

16. Hwang HW and Mendell JT: MicroRNAs in cell proliferation, cell death, and tumorigenesis. Br J Cancer 96 (Suppl 1): R40-R44, 2007.

17. Bartel DP: MicroRNAs: Genomics, biogenesis, mechanism, and function. Cell 116: 281-297, 2004.

18. Xiao Y, Zhang S, Li Q, Liu Z, Mai W, Chen W, Lei J and Hu H: miR-219a-5p Ameliorates hepatic ischemia/reperfusion injury via impairing TP53BP2. Dig Dis Sci 64: 2177-2186, 2019.

19. Xing Y, Li J, Li SP, Xi J, Ma N, Liu L, Wang JS and Cai JZ: MiR-27a-5p regulates apoptosis of liver ischemia-reperfusion injury in mice by targeting Bach1. J Cell Biochem 119: 10376-10383, 2018.

20. Yuan J and Zhang Y: Sevoflurane reduces inflammatory factor expression, increases viability and inhibits apoptosis of lung cells in acute lung injury by microRNA-34a-3p upregulation and STAT1 downregulation. Chem Biol Interact 322: 109027 , 2020.

21. Xu C, Niu JJ, Zhou JF and Wei YS: MicroRNA-96 is responsible for sevoflurane-induced cognitive dysfunction in neonatal rats via inhibiting IGF1R. Brain Res Bull 144: 140-148, 2019.

22. Zhao YB, Zhao J, Zhang LJ, Shan RG, Sun ZZ, Wang K, Chen JQ and Mu JX: MicroRNA-370 protects against myocardial ischemia/reperfusion injury in mice following sevoflurane anesthetic preconditioning through PLIN5-dependent PPAR signaling pathway. Biomed Pharmacother 113: 108697, 2019.

23. Tan DX, Chen XX, Bai TZ, Zhang J and Li ZF: Sevoflurane up-regulates microRNA-204 to ameliorate myocardial ischemia/reperfusion injury in mice by suppressing Cotl1. Life Sci 259: 118162, 2020. 
24. Liao X, Zhou S, Zong J and Wang Z: Sevoflurane exerts protective effects on liver ischemia/reperfusion injury by regulating NFKB3 expression via miR-9-5p. Exp Ther Med 17: 2632-2640, 2019.

25. Livak KJ and Schmittgen TD: Analysis of relative gene expression data using real-time quantitative PCR and the 2(-Delta Delta C(T)) method. Methods 25: 402-408, 2001

26. Li XF, Wang ZQ, Li LY, Zhao GQ and Yu SN: Downregulation of the long noncoding RNA MBNL1-AS1 protects sevoflurane-pretreated mice against ischemia-reperfusion injury by targeting KCNMA1. Exp Mol Med 50: 115, 2018.

27. Shi CX, Ding YB, Jin FYJ, Li T, Ma JH, Qiao LY, Pan WZ and Li KZ: Effects of sevoflurane post-conditioning in cerebral ischemia-reperfusion injury via TLR4/NF- $\kappa \mathrm{B}$ pathway in rats. Eur Rev Med Pharmacol Sci 22: 1770-1775, 2018.

28. Hao YL, Fang HC, Zhao HL, Li XL, Luo Y, Wu BQ, Fu MJ, Liu W, Liang JJ and Chen XH: The role of microRNA-1 targeting of MAPK3 in myocardial ischemia-reperfusion injury in rats undergoing sevoflurane preconditioning via the PI3K/Akt pathway. Am J Physiol Cell Physiol 315: C380-C388, 2018.

29. Qiao SG, Sun Y, Sun B, Wang A, Qiu J, Hong L, An JZ, Wang C and Zhang HL: Sevoflurane postconditioning protects against myocardial ischemia/reperfusion injury by restoring autophagic flux via an NO-dependent mechanism. Acta Pharmacol Sin 40 35-45, 2019.

30. Ming N, Na HST, He JL, Meng QT and Xia ZY: Propofol alleviates oxidative stress via upregulating lncRNA-TUG1/Brg1 pathway in hypoxia/reoxygenation hepatic cells. J Biochem 166 : 415-421, 2019.

31. Wenlan L, Zhongyuan X, Shaoqing L, Liying Z, Bo Z and Min L: MiR-34a-5p mediates sevoflurane preconditioning induced inhibition of hypoxia/reoxygenation injury through STX1A in cardiomyocytes. Biomed Pharmacother 102: 153-159, 2018.

32. Hao W, Zhao ZH, Meng QT, Tie ME, Lei SQ and Xia ZY: Propofol protects against hepatic ischemia/reperfusion injury via miR-133a-5p regulating the expression of MAPK6. Cell Biol Int 41: 495-504, 2017.

33. Xiong L, Yu KH and Zhen SQ: MiR-93 blocks STAT3 to alleviate hepatic injury after ischemia-reperfusion. Eur Rev Med Pharmacol Sci 22: 5295-5304, 2018.

34. Wu Y, Gu C and Huang X: Sevoflurane protects against hepatic ischemia/reperfusion injury by modulating microRNA-200c regulation in mice. Biomed Pharmacother 84: 1126-1136, 2016.

35. Li S, Zhang J, Wang Z, Wang T, Yu Y, He J, Zhang H, Yang T and Shen Z: MicroRNA-17 regulates autophagy to promote hepatic ischemia/reperfusion injury via suppression of signal transductions and activation of transcription-3 expression. Liver Transpl 22: 1697-1709, 2016.

36. Tian J, Zhang H, Mu L, Wang M, Li X, Zhang X, Xie E, Ma M, Wu D and Du Y: The miR-218/GAB2 axis regulates proliferation, invasion and EMT via the PI3K/AKT/GSK-3 $\beta$ pathway in prostate cancer. Exp Cell Res 394: 112128, 2020.

37. Shi L, Sun X, Zhang J, Zhao C, Li H, Liu Z, Fang C, Wang X, Zhao C, Zhang X, et al: Gab2 expression in glioma and its implications for tumor invasion. Acta Oncol 52: 1739-1750, 2013

38. Liu T, Zhang Q, Mo W, Yu Q, Xu S, Li J, Li S, Feng J, Wu L, $\mathrm{Lu} X$, et al: The protective effects of shikonin on hepatic ischemia/reperfusion injury are mediated by the activation of the PI3K/Akt pathway. Sci Rep 7: 44785, 2017.

39. Li S, Yi Z, Deng M, Scott MJ, Yang C, Li W, Lei Z, Santerre NM, Loughran $\mathrm{P}$ and Billiar TR: TSLP protects against liver I/R injury via activation of the PI3K/Akt pathway. JCI Insight 4 e129013, 2019.

40. Zuniga J, Venegas F, Villarreal M, Núñez D, Chandía M, Valenzuela R, Tapia G, Varela P, Videla LA and Fernández V: Protection against in vivo liver ischemia-reperfusion injury by n-3 long-chain polyunsaturated fatty acids in the rat. Free Radic Res 44:854-863, 2010.

41. Mardones M, Valenzuela R, Romanque P, Covarrubias N Anghileri F, Fernández V, Videla LA and Tapia G: Prevention of liver ischemia reperfusion injury by a combined thyroid hormone and fish oil protocol. J Nutr Biochem 23: 1113-1120, 2012.

42. Zhang J, Wang C, Yu S, Luo Z, Chen Y, Liu Q, Hua F, Xu G and Yu P: Sevoflurane postconditioning protects rat hearts against ischemia-reperfusion injury via the activation of PI3K/AKT/mTOR signaling. Sci Rep 4: 7317, 2014.

43. Takemori K, Kobayashi K and Sakamoto A: Expression of pulmonary vasoactive factors after sevoflurane anaesthesia in rats: A quantitative real-time polymerase chain reaction study. Br J Anaesth 100: 190-194, 2008.
44. Yu P, Zhang J, Yu S, Luo Z, Hua F, Yuan L, Zhou Z, Liu Q, Du X, Chen $S$, et al: Protective effect of sevoflurane postconditioning against cardiac ischemia/reperfusion injury via ameliorating mitochondrial impairment, oxidative stress and rescuing autophagic clearance. PLoS One 10: e0134666, 2015.

45. Mikrou A, Kalimeris KA, Lilis I, Papoutsidakis N, Nastos K, Papadaki H, Kostopanagiotou GG and Zarkadis IK: Molecular studies of the immunological effects of the sevoflurane preconditioning in the liver and lung in a rat model of liver ischemia/reperfusion injury. Mol Immunol 72: 1-8, 2016

46. Huang G, Hao F and Hu X: Downregulation of microRNA-155 stimulates sevoflurane-mediated cardioprotection against myocardial ischemia/reperfusion injury by binding to SIRT1 in mice. J Cell Biochem 120: 15494-15505, 2019.

47. Zhu H, Wang X and Chen S: Downregulation of miR-218-5p protects against oxygen-glucose deprivation/reperfusion-induced injuries of $\mathrm{PC} 12$ cells via upregulating $\mathrm{N}$-myc downstream regulated gene 4 (NDRG4). Med Sci Monit 26: e920101, 2020.

48. Su SS, Li BP, Li CL, Xiu FR, Wang DY and Zhang FR: Downregulation of MiR-218 can alleviate high-glucose-induced renal proximal tubule injury by targeting GPRC5A. Biosci Biotechnol Biochem 84: 1123-1130, 2020.

49. $\mathrm{Li} \mathrm{L}$ and Zhao G: Downregulation of microRNA-218 relieves neuropathic pain by regulating suppressor of cytokine signaling 3 . Int J Mol Med 37: 851-858, 2016

50. Starkey Lewis PJ, Dear J, Platt V, Simpson KJ, Craig DG, Antoine DJ, French NS, Dhaun N, Webb DJ, Costello EM, et al: Circulating microRNAs as potential markers of human drug-induced liver injury. Hepatology 54: 1767-1776, 2011.

51. Rao J, Qian X, Li G, Pan X, Zhang C, Zhang F, Zhai Y, Wang X and Lu L: ATF3-mediated NRF2/HO-1 signaling regulates TLR4 innate immune responses in mouse liver ischemia/reperfusion injury. Am J Transplant 15: 76-87, 2015.

52. Park SW, Chen SW, Kim M, Brown KM, D'Agati VD and Lee HT: Protection against acute kidney injury via $\mathrm{A}(1)$ adenosine receptor-mediated Akt activation reduces liver injury after liver ischemia and reperfusion in mice. J Pharmacol Exp Ther 333: 736-747, 2010

53. Adams SJ, Aydin IT and Celebi JT: GAB2-a scaffolding protein in cancer. Mol Cancer Res 10: 1265-1270, 2012.

54. Li Z, Zhao F, Cao Y, Zhang J, Shi P, Sun X, Zhang F and Tong L: DHA attenuates hepatic ischemia reperfusion injury by inhibiting pyroptosis and activating PI3K/Akt pathway. Eur J Pharmacol 835: 1-10, 2018.

55. Li H, Chen O, Ye Z, Zhang R, Hu H, Zhang N, Huang J, Liu W and Sun $X$ : Inhalation of high concentrations of hydrogen ameliorates liver ischemia/reperfusion injury through $\mathrm{A}_{2 \mathrm{~A}}$ receptor mediated PI3K-Akt pathway. Biochem Pharmacol 130: 83-92, 2017.

56. Mu L, Guan B, Tian J, Li X, Long Q, Wang M, Wang W, She J, $\mathrm{Li}$ X, Wu D and Du Y: MicroRNA218 inhibits tumor angiogenesis of human renal cell carcinoma by targeting GAB2. Oncol Rep 44: 1961-1970, 2020.

57. Marchi S, Giorgi C, Suski JM, Agnoletto C, Bononi A, Bonora M, De Marchi E, Missiroli S, Patergnani S, Poletti F, et al: Mitochondria-ros crosstalk in the control of cell death and aging. J Signal Transduct 2012: 329635, 2012.

58. Quoilin C, Mouithys-Mickalad A, Lecart S, Fontaine-Aupart MP and Hoebeke M: Evidence of oxidative stress and mitochondrial respiratory chain dysfunction in an in vitro model of sepsis-induced kidney injury. Biochim Biophys Acta 1837: 1790-1800, 2014.

59. Ortiz M, Soto-Alarcon SA, Orellana P, Espinosa A, Campos C, López-Arana S, Rincón MA, Illesca P, Valenzuela R and Videla LA: Suppression of high-fat diet-induced obesity-associated liver mitochondrial dysfunction by docosahexaenoic acid and hydroxytyrosol co-administration. Dig Liver Dis 52: 895-904, 2020.

60. Okatani Y, Wakatsuki A, Enzan H and Miyahara Y: Edaravone protects against ischemia/reperfusion-induced oxidative damage to mitochondria in rat liver. Eur J Pharmacol 465: 163-170, 2003.

61. Mukhopadhyay P, Horvath B, Zsengeller Z, Bátkai S, Cao Z, Kechrid M, Holovac E, Erdèlyi K, Tanchian G, Liaudet L, et al: Mitochondrial reactive oxygen species generation triggers inflammatory response and tissue injury associated with hepatic ischemia-reperfusion: Therapeutic potential of mitochondrially targeted antioxidants. Free Radic Biol Med 53: 1123-1138, 2012. 